\title{
Evaluation of lower urinary tract symptoms in multiple sclerosis patients: Review of the literature and current guidelines
}

\author{
Shachar Moshe Aharony, MD;1 Ornella Lam, MD;2 Jacques Corcos, MD² \\ Urology Service, Rabin Medical Centre, Beilinson Hospital, Pteach-Tiqva, Israel; '2Department of Urology, Jewish General Hospital, McGill University, Montreal, QC, Canada
}

Cite as: Can Urol Assoc J 2017;11 (1-2):61-4. http://dx.doi.org/10.5489/cuaj.4058

See related commentary on page 65 .

Read a followup of this article, "Treatment of lower urinary tract symptoms in multiple sclerosis patients," in next month's online issue of CUAJ (www.cuaj.ca).

\section{Abstract}

Multiple sclerosis (MS) is a unique neurological disease with a broad spectrum of clinical presentations that are time- and disease course-related. MS plaque location (intracranial and/or spinal) is a key feature in the pathophysiology of disease-related lower urinary tract symptoms (LUTS). The prevalence of these symptoms in MS patients is very high, with nearly $90 \%$ of them experiencing some degree of voiding dysfunction and/or incontinence. LUTS rarely present as primary MS manifestations and usually appear 6-8 years after the initial diagnosis. Symptom severity usually correlates with the disability status of patients.

Patient assessment comprises clinical and advanced investigations. Each patient should be evaluated uniquely, after taking into account his/her symptoms, disease course and length, comorbidities, physical status, and medications. Basic investigation includes detailed history-taking, physical examination, and post-void residual volume measurement. Advanced evaluation consists of imaging and specific testing, with pivotal importance on urodynamic study.

\section{Introduction}

Multiple sclerosis (MS) is a unique, inflammatory central nervous system (CNS) disease with a broad spectrum of clinical presentations that are time- and disease progression-related. It usually affects young adults, with a female predominance of 3:1. Men are more likely to develop symptoms at a slightly older age, with a more rapidly progressing disease course.

Although urological symptoms as first presentation of MS are rare (3-10\%), almost two-thirds of MS patients will suffer from moderate to severe urinary disturbances related to their disease. These can result in significant morbidity and impairment of their quality of life (QOL). ${ }^{1}$ Urologists must have a thorough knowledge of the disease process, as we have discussed in a prior article, ${ }^{2}$ to tailor the right evaluation tools to each specific case.

\section{Pathophysiology of lower urinary tract symptoms (LUTS) in MS}

MS plaques can be found anywhere in the CNS, including the spinal cord. Their exact location will profile unique features of lower urinary tract dysfunction (LUTD). Intracranial lesions occur in up to $90 \%$ of MS patients nearly everywhere along the white matter. Lesions in cortical regions related to urinary tract regulation (medial prefrontal cortex, insula, and pons) are thought to be the cause of detrusor overactivity (DO). Spinal cord, and particularly suprasacral lesions, are common in MS patients, as described by Oppenheimer. ${ }^{3}$ The prevalence of cervical cord plaques is almost $80 \%$ in these cases, predominantly in the lateral corticospinal (pyramidal) and reticulospinal tracts. Lumbar and dorsal cord involvement is less frequent $(40 \%$ and $18 \%$, respectively). Suprasacral spinal lesions may cause DO by impacting the descending inhibition of bladder contraction. On the other hand, damage to the reticulospinal tracts may lead to detrusor-sphincter-dyssynergia (DSD). ${ }^{4}$ Sacral cord lesions are less common (18-60\%) and their role in LUTD is still questionable. Plaques in efferent or afferent pathways may impair emptying and urinary retention. ${ }^{5}$ Although $63 \%$ of patients with sacral lesions show detrusor hypocontractility, only $5 \%$ present bladder areflexia. ${ }^{6}$

\section{Prevalence of LUTS in MS}

LUTS are common in MS patients. Based on the North American Research Committee on Multiple Sclerosis Registry, a large survey of more than 9700 MS patients, ${ }^{7} 65 \%$ reported moderate to severe urinary complaints. Nocturia, followed by urinary urgency and frequency were the most prevalent signs. Urinary incontinence and poor bladder emptying were noted less frequently. Table 1 summarizes the data on and prevalence of LUTS in these patients. 
Aharony et al.

\begin{tabular}{lccccc}
\hline Table 1. Percentage of MS patients experiencing LUTS & & & \\
\hline Study & No. of patients & Urgency & Frequency & Incontinence & Hesitancy \\
\hline Miller et al ${ }^{36}$ & 321 & 60 & 50 & 36 & 33 \\
Bradley et al $^{37}$ & 90 & 86 & 60 & & 28 \\
Hennessey et al $^{38}$ & 191 & 71 & 76 & 19 & 20 \\
Borello-France et al $^{39}$ & 133 & 61 & 71 & 83 & 58 \\
Ukkonen et al $^{40}$ & 24 & 83 & 54 & 75 & 38 \\
Quarto et al $^{31}$ & 107 & 61 & 83 & 32 & \\
\hline Modified from Fernández $^{28}$ and Quarto et al. & & \\
\hline
\end{tabular}

Urinary symptoms rarely represent the first manifestations of MS (up to 10\%) and usually appear 6-8 years after the initial diagnosis. ${ }^{8}$ The presence or absence of symptoms is an unreliable indicator of voiding dysfunction extent. Although most symptomatic patients will manifest some objective features on urodynamic study (UDS), lack of symptoms corresponds poorly with this test. ${ }^{1}$ Storage symptoms correlate well with the Expanded Disability Status Scale (EDSS) ${ }^{9}$ and pyramidal tract involvement; however, their association with voiding symptoms is much weaker. ${ }^{10}$

LUTS in MS may be influenced by gender and age. While their exact impact on patient symptoms has not been thoroughly investigated, cumulative action of other diseases is possible, eliciting bladder dysfunction - benign prostate obstruction, pelvic relaxation, or stress urinary incontinence (SUI). There appears to be no significant relationship between the overall incidence of symptoms and gender. However, men with MS have a higher prevalence of voiding symptoms and complications, especially after age $50 .{ }^{11}$

\section{Evaluation of LUTS in MS}

MS is characterized by heterogeneous clinical presentation and evolution. Patient profiles vary greatly. Although most patients urinate by themselves, they may suffer from overactive bladder $(\mathrm{OAB})$ symptoms or recurrent urinary tract infections (UTIs). Other common complaints are voiding and emptying difficulties with a smaller group of patients who cannot void at all.
Evaluation has to be uniquely tailored for every patient after taking into account his/her symptoms, disease course and length, comorbidities, physical status, and medications. However, complete history, including QOL assessment, physical examination, and urine culture and analysis with post-void residual (PVR) measurements, should be obtained systematically for all patients. More advanced evaluation, including UDS, may be reserved for cases where the results may change the treatment regimen chosen.

At present, international management guidelines, developed by the International Consultation on Incontinence $(\mathrm{ICI})^{12}$ and the European Association of Urology (EAU), ${ }^{13}$ exist for neurogenic LUTD (NLUTD) in general, but not specifically for MS patients. Unlike spinal cord injury (SCI), MS patients, even with detrusor external sphincter dyssynergia (DESD) and neurogenic detrusor overactivity (NDO), rarely suffer from upper urinary tract (UUT) deterioration. ${ }^{1,11}$ Therefore, active and invasive evaluation of NLUTD patients, as proposed by these guidelines, does not seem fully applicable to the MS population.

Several expert European national panels published their own management consensus on MS. ${ }^{14-20}$ Unfortunately, the scarcity of good-quality studies assessing the efficacy and safety of various treatments in MS lessens their levels of evidence. There are considerable differences between these guidelines, depending on the target population to whom they are addressed. They vary slightly if they have been laid down for general practitioners, rehabilitation specialists and neurologists, ${ }^{15,17,19,20}$ urologists, ${ }^{16}$ or neurourologists. ${ }^{18}$

Table 2. Which MS patients should be screened for LUTS?

\begin{tabular}{|c|c|c|c|}
\hline & Italy ${ }^{20}$ & France $^{15}$ & Belgium $^{17}$ \\
\hline EDSS & $\geq 3$ & $\geq 6$ & Significant impact on $\mathrm{OOL}$ \\
\hline MS duration & Long-term & $\mathrm{N} / \mathrm{A}$ & $\geq 15$ years \\
\hline Gender and age & Male $\geq 50$ years & Male $\geq 55$ years & Male $\geq 50$ years \\
\hline PVR & $\geq 100 \mathrm{cc}$ or $1 / 3 \mathrm{BC}$ & $\geq 100 \mathrm{cc}$ & $\geq 100-150$ cc \\
\hline Recurrent UTIs & Yes & Associated with fever or lumbar pain & Yes \\
\hline Abnormal ultrasound findings* & Yes & Yes & Yes \\
\hline $\begin{array}{l}\text { Failure of conservative } \\
\text { treatment }\end{array}$ & $\begin{array}{l}\text { Medications and } \\
\text { catheterizations }\end{array}$ & Medications & Medication and catheter bypass \\
\hline Other & Impaired $\mathrm{Cr}$ blood levels & Immunosuppression & $\begin{array}{l}\text { Incontinence } \\
\text { More than } 2 \text { risk factors }{ }^{*}\end{array}$ \\
\hline
\end{tabular}

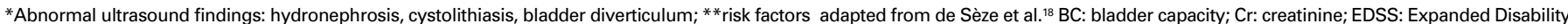
Status Scale; LUTS: lower urinary tract symptoms; MS: multiple sclerosis; N/A: not available; PVR: post-void residual; QOL: quality of life; UTI: urinary tract infection. 
While all groups advocate urological evaluation of symptomatic patients, there is no consensus regarding the necessity of urinary tract evaluation in MS patients without LUTS. Litwiller et al ${ }^{1}$ showed that even asymptomatic MS patients can suffer from LUTS. This has led a few groups to develop specific screening questionnaires, ${ }^{15,21}$ while others based their profiling on combination of patients' properties with different test results. ${ }^{18,20}$ Table 2 lists the recommendations made for different screening criteria.

Critical analysis of different guidelines ${ }^{15-21}$ has resulted in dividing MS-related LUTS evaluation into basic and advanced testing.

\section{Basic testing}

History-taking should include detailed clinical background with emphasis on symptoms of urgency, frequency, incontinence (stress- and urgency-related), hesitancy, retention, and nocturia. LUTS may disturb QOL as much as motor disability does in MS patients. ${ }^{22}$ Therefore, non-specific QOL instruments, such as King's Health Questionnaire, ${ }^{23} \mathrm{OAB}$ symptom scores, ${ }^{24}$ and condition-specific Qualiveen, ${ }^{25}$ are useful in evaluating daily impact and disease progression over time.

Physical examination should include appraisal of motor and sensory dysfunctions of the lower extremities, pelvic and sacral dermatomes. Neurologist review of physical examination may add important information. Pontine signs (dizziness, visual disturbances) may predict the future presense of NDO, while cerebellar signs may indicate DSD. ${ }^{26}$ Pyramidial impairment of the lower limbs reflects the extent of spinal involvment and correlates with LUTS severity. ${ }^{27}$ Digital rectal examination, pelvic measurements, and stress incontinence maneuvers may be needed to ascertain benign prostatic enlargement, malignancy, constipation, pelvic organ prolapse, and SUI. ${ }^{1,28}$

PVR volume should be evaluated, either by catheterization or preferably by bladder scan. MS patients have larger PVR volumes than their control counterparts, ${ }^{29,30}$ and mean PVR is greater in MS patients with recurrent UTIs than in those without. ${ }^{31}$

Other important evaluation tools are three-day voiding or catheterization diaries, urine analysis, and culture.

\section{Advanced testing}

Advanced testing is not indicated for all patients. Generally, such assessment is reserved for cases where the chosen therapy could be changed based on the results.

\section{Imaging}

There is no consensus on imaging as a screening tool or confined to symptomatic patients at risk of UUT detrioration. Most guidelines recommend ultrasound in which hydro- nephrosis, cystolithiasis, and bladder diverticulum are considered to be UUT risk factors. ${ }^{15,17,20}$

Multichannel UDS and especially video-UDS are highly recommended by the EAU for neurogenic-related LUTS assessment (Grade A recommendation), ${ }^{13}$ as repeated measurements can influence clinical decision-making (Grade $\mathrm{C}$ recommendation). However, these recommendations are not MS-specific.

In recent years, recommendations on UDS for LUTS evaluation in MS patients have changed drastically. de Sèze et al ${ }^{18}$ proposed UDS for all symptomatic patients. However, more recent recommendations ${ }^{15-17,19,20}$ and the fifth $\mathrm{ICl}^{12}$ propose limits to UDS, suggesting urodynamic evaluation only in patients at risk of UUT deterioration or failure of initial, conservative treatment. However, UDSs are still recommended before any intravesical or surgical intervention. Although Blaivas et $\mathrm{a}^{132}$ showed that $73 \%$ of MS patients without UDS were treated inappropriately, it remains an evaluation tool mainly employed by neurourologists. Wiedemann et al ${ }^{33}$ tried to define risk profiling necessitating UDS. EDSS $\geq 6.5 \mathrm{MS}$ subtypes other than relapsing-remitting and the use of more than one incontinence pad per day were found to increase the likelihood of abnormal UDS findings.

Five major urodynamic patterns can be seen in MS: ${ }^{34}$

- NDO without DSD

- NDO with DSD

- NDO with impaired contractility

- Detrusor underactivity (DU)

- Normal function

In a meta-analysis by Litwiller et al, ${ }^{1} 62 \%$ had NDO, 25\% had signs of DSD, $20 \%$ had DU, and $10 \%$ were normal on examination. Comparing MS patients with signs of NDO on UDS to patients with idiopathic DO revealed significant changes. MS patients had smaller bladder capacity with higher PVR volume and increased DO amplitude. Whether it is due to heightened bladder outlet resistance (DESD, for example) or is neurogenically mediated is still debatable. ${ }^{30}$ Later work by the same authors ${ }^{35}$ focused on the role of DSD in urinary dysfunction among women with MS. In a fouryear followup period, no signs of UUT deterioration were documented in 143 patients. The authors concluded that the lack of significant detrusor pressure elevation in the DESD group accounts for the relatively low incidence of UUT damage in MS patients compared to $\mathrm{SCl}$ cases. More than that, no linkage was confirmed between MS subtype, time since diagnosis, patient age, and the presence of DESD. As MS is a fluctuating disease with exacerbations and remissions, UDS depicts urinary tract function at certain time points. Although improvement may occur, DSD is rarely resolved. ${ }^{1}$

Cystoscopy is not mentioned routinely in the above guidelines. It is usually reserved for the evaluation of recurrent UTIs, cystolithiasis, bladder outlet obstruction, and incontinence. 


\section{Conclusion}

Most of the management consensuses (Grade D recommendations) discussed above are intended for urologists and general practitioners. Proposed diagnosis and followup algorithms differ as well, reflecting the diversity of clinicians' opinions on MS treatments, with variance between healthcare systems and the availability of different specialists. Urologists would be wise to adopt this consensus, as no one optimal management protocol suits all. They need to tailor specific evaluation protocols based on their experience and armamentarium to make the exact diagnosis and propose the best possible treatment.

Competing interests: Dr. Corcos has been an advisor for Allergan, Astellas, and Pfizer; a speaker for Allergan and Duchesnay; has received payment and honoraria from Astellas; and has participated in clinical trials for Allergan and Ipsen. The remaining authors report no competing personal or financial interests.

This paper has been peer-reviewed.

\section{References}

1. Litwiller SE, Frohman EM, Zimmern PE. Multiple sclerosis and the urologist. J Urol 1999;161:743-57. https://doi.org/10.1016/S0022-5347(01)61760-9

2. Aharony S, Lam 0, Lapierre Y, et al. Multiple sclerosis (MS) for the urologist: What should urologists know about MS? Neurourol Urodyn 2016;35:174-9. https://doi.org/10.1002/nau.22713

3. Oppenheimer D. The cervical cord in multiple sclerosis. Neuropathol Appl Neurobiol 1978;4:151-62. https://doi.org/10.1111/i.1365-2990.1978.tb00555.x

4. Blaivas J, Barbalias $G$. Detrusor-external sphincter dyssynergia in men with multiple sclerosis: An ominous urologic condition. J Urol 1984;131:91-4.

5. Stoffel JT. Contemporary management of the neurogenic bladder for multiple sclerosis patients. Urol Clin North Am 2010;37:547-57. https://doi.org/10.1016/j.ucl.2010.06.003

6. Mayo ME, Chetner MP. Lower urinary tract dysfunction in multiple sclerosis. Urology 1992;39:67-70. https://doi.org/10.1016/0090-4295(92)90045-X

7. Marrie R, Cutter $G$, Tyry T, et al. Disparities in the management of multiple sclerosis-related bladder symptoms. Neurology 2007;68:1971-8. https://doi.org/10.1212/01.wnl.0000264416.53077.8b

8. PHADKE JG. Clinical aspects of multiple sclerosis in north-east Scotland with particular reference to its course and prognosis. Brain 1990;113:1597-628. https://doi.org/10.1093/brain/113.6.1597

9. DasGupta R, Fowler C. Bladder, bowel, and sexual dysfunction in multiple sclerosis: Management strategies. Drugs 2003;63:153. https://doi.org/10.2165/00003495-200363020-00003

10. Araki I, Matsui M, Ozawa K, et al. Relationship between urinary symptoms and disease-related parameters in multiple sclerosis. J Neurol 2002; 249:1010-5. https://doi.org/10.1007/s00415-002-0775-4

11. Koldewijn EL, Hommes OR, Lemmens WA, et al. Relationship between lower urinary tract abnormalities and disease-related parameters in multiple sclerosis. J Urol 1995;154:169-73. https://doi.org/10.1016/ S0022-5347(01)67258-6

12. Abrams PCL, Khoury S, Wein A. Incontinence. 5th Edition. Arnhem: European association of urology; 2013.

13. Groen J, Pannek J, Castro Diaz D, et al. Summary of European Association of Urology (EAU) guidelines on neuro-urology. Eur Urol 2016;69:324-33. https://doi.org4032/10.1016/i.eururo.2015.07.071

14. Abrams $P$, Cardozo L, Fall M, et al. Standardization Subcommittee of the International Continence Society. The standardization of terminology of lower urinary tract function: Report from the Standardization Subcommittee of the International Continence Society. Neurourol Urodyn 2002;21:167-78. https://doi.org/10.1002/nau.10052

15. Amarenco $G$, Chartier-Kastler $E$, Denys $P$, et al. First-line urological evaluation in multiple sclerosis: Validation of a specific decision-making algorithm. Mult Scler 2013;19:1931-7. https://doi.org/10.1177/1352458513489758

16. Cetinel B, Tarcan T, Demirkesen 0 , et al. Management of lower urinary tract dysfunction in multiple sclerosis: A systematic review and Turkish consensus report. Neurourol Urodyn 2013;32:1047-57. https://doi.org/10.1002/nau.22374
17. De Ridder D, Van der Aa F, Debruyne J, et al. Consensus guidelines on the neurologist's role in the management of neurogenic lower urinary tract dysfunction in multiple sclerosis. Clin Neurol Neurosurg 2013;115:2033-40. https://doi.org/10.1016/i.clineuro.2013.06.018

18. de Sèze M, Ruffion A, Denys $P$, e al. The neurogenic bladder in multiple sclerosis: Review of the literature and proposal of management guidelines. Mult Scler 2007;13:915-28. htrps://doi.org/10.1177/1352458506075651

19. Fowler C, Panicker J, Drake M, et al. A UK consensus on the management of the bladder in multiple sclerosis. J Neurol Neurosurg Psychiatry 2009;80:470-7. htrps://doi.org/10.1136/innp.2008.159178

20. Ghezzi A, Carone R, Del Popolo G, et al. Recommendations for the management of urinary disorders in multiple sclerosis: A consensus of the Italian Multiple Sclerosis Study Group. Neurol Sci 201 1;32:1223-31. https://doi.org/10.1007/s10072-011-0794-y

21. Burks J, Chancellor M, Bates D, et al. Development and validation of the actionable bladder symptom screening tool for multiple sclerosis patients. Int J MS Care 2013;15:182-92. https://doi.org/10.7224/1537-2073.2012-049

22. Mahajan $S$, Patel $P$, Marrie R. Under treatment of overactive bladder symptoms in patients with multiple sclerosis: An ancillary analysis of the NARCOMS Patient Registry. J Urol 2010;183:1432-7. https://doi.org/10.1016/i.juro.2009.12.029

23. Kelleher C, Cardozo L, Khullar $V$, et al. A new questionnaire to assess the quality of life of urinary incontinent women. Br J Obstet Gynaecol 1997;104:1374-9. https://doi.org/10.1111/i.1471-0528.1997.tb1 1006.x

24. Homma Y, Yoshida M, Seki N, et al. Symptom assessment tool for overactive bladder syndrome - overactive bladder symptom score. Urology 2006;68:318-23. https://doi.org/10.1016/i.urology.2006.02.042

25. Bonniaud V, Bryant D, Parratte B, et al. Qualiveen: A urinary disorder-specific instrument for use in clinical trials in multiple sclerosis. Arch Phys Med Rehabil 2006; 87:1661-3. https://doi.org/10.1016/i. apmr.2006.08.345

26. Araki I, Matsui M, Ozawa K, et al. Relationship of bladder dysfunction to lesion site in multiple sclerosis. J Urol 2003;169:1384-7. https://doi.org/10.1097/01.ju.0000049644.27713.c8

27. Betts $C D, D^{\prime}$ Mellow $M$, Fowler $C J$. Urinary symptoms and the neurological features of bladder dysfunction in multiple sclerosis. J Neurol Neurosurg Psychiatry 1993;56:245-50. https://doi.org/10.1136/ jnnp.56.3.245

28. Fernández 0 . Mechanisms and current treatments of urogenital dysfunction in multiple sclerosis. J Neurol 2002;249:1-8. https://doi.org/10.1007/PL00007835

29. Gray R, Wagg A, Malone-Lee J. Differences in detrusor contractile function in women with neuropathic and idiopathic detrusor instability. Br J Urol 1997;80:222-6. https://doi.org/10.1046/i.1464410X.1997.00259.x

30. Lemack GE, Frohman EM, Zimmern PE, et al. Urodynamic distinctions between idiopathic detrusor overactivity and detrusor overactivity secondary to multiple sclerosis. Urology 2006;67:960-4. https://doi.org/10.1016/i.urology.2005.11.061

31. Quarto $G$, Autorino R, Gallo A, et al. Quality of life in women with multiple sclerosis and overactive bladder syndrome. Int Urogynecol J Pelvic Floor Dysfunct 2007;18:189-94. https://doi.org/10.1007/s00192-006-0131-9

32. Blaivas IG, Holland NJ, Giesser B, et al. Multiple sclerosis bladder: Studies and care. Ann NY Acad Sci 1984;436:328-45. https://doi.org/10.1111/i.1749-6632.1984.tb14804.x

33. Wiedemann $A$, Kaeder $M$, Greulich $W$, et al. Which clinical risk factors determine a pathological urodynamic evaluation in patients with multiple sclerosis? An analysis of 100 prospective cases. World J Urol 2013;31:229-33. https://doi.org/10.1007/s00345-011-0820-y

34. Abrams $P$, Cardozo L, Fall $M$, et al. The standardization of terminology in lower urinary tract function: Report from the standardization subcommittee of the International Continence Society. Urology 2003;61:37-49. https://doi.org/10.1016/S0090-4295(02)02243-4

35. Lemack GE, Frohman E, Ramnarayan P. Women with voiding dysfunction secondary to bladder outlet dyssynergia in the setting of multiple sclerosis do not demonstrate significantly elevated intravesical pressures. Urology 2007;69:893-7. https://doi.org/10.1016/i.urology.2007.01.076

36. Miller H, Simpson CA, Yeates WK. Bladder dysfunction in multiple sclerosis. Br Med J 1965;1:1265. https://doi.org/10.1136/bmi.1.5445.1265

37. Bradley WE, Logothetis JL, Timm GW. Cystometric and sphincter abnormalities in multiple sclerosis. Neurology 1973;23:1131-9. https://doi.org/10.1212/WNL.23.10.1131

38. Hennessey A, Robertson N, Swingler R, et al. Urinary, fecal, and sexual dysfunction in patients with multiple sclerosis. J Neurol 1999;246:1027-32. htrps://doi.org/10.1007/s004150050508

39. Borello-France $D$, Leng $W, O^{\prime}$ Leary $M$, et al. Bladder and sexual function among women with multiple sclerosis. Mult Scler 2004;10:455-61. https://doi.org/10.1191/1352458504ms1060oa

40. Ukkonen $M$, Elovaara I, Dastidar $\mathrm{P}$, et al. Urodynamic findings in primary progressive multiple sclerosis are associated with increased volumes of plaques and atrophy in the central nervous system. Acta Neurologica Scandinavica 2004;109:100-5. https://doi.org/10.1034/i.1600-0404.2003.00184.x

Correspondence: Dr. Shachar Moshe Aharony, Urology Service, Rabin Medical Centre, Beilinson Hospital, Pteach-Tiqva, Israel; shaharony@gmail.com 